PROCEEDINGS OF THE

AMERICAN MATHEMATICAL SOCIETY

Volume 137, Number 12, December 2009, Pages 4187-4197

S 0002-9939(09)09995-X

Article electronically published on July 14, 2009

\title{
LOCALIZABLE OPERATORS AND THE CONSTRUCTION OF LOCALIZED FRAMES
}

\author{
FUMIKO FUTAMURA
}

(Communicated by Michael T. Lacey)

\begin{abstract}
We introduce the notion of localizable operators with respect to frames and prove the boundedness of such operators on families of Banach spaces. This generalizes previous results for specific operators, such as pseudodifferential operators on modulation spaces. We also use this notion to provide sufficient conditions for the construction of frames which have the localization property.
\end{abstract}

\section{INTRODUCTION}

Given a collection of compact normal operators on a separable Hilbert space $\mathcal{H}$, it is often desirable, but not always possible, to find a single basis that diagonalizes every operator in the class. Thus, instead of diagonalizing the operators simultaneously, it is often useful to find a basis or, more generally, a pair of frames that almost diagonalizes all the operators in the sense that the matrix representation has fast off-diagonal decay for each operator in the class.

For a finite collection of symmetric matrices in finite dimensions, there is a technique for finding a basis that almost diagonalizes such a collection. This technique, known as approximate joint diagonalization, was studied by both mathematicians and engineers for blind source separation algorithms (see, for example, 7]).

For an infinite collection of operators in infinite dimensions, only a few specific examples have been studied and resolved. For example, Frazier and Jawerth proved that by choosing a well-behaved orthonormal wavelet frame, we can almost diagonalize the Calderón-Zygmund operators of the reduced David-Journé class [10, 8. These operators are a generalization of pseudodifferential operators and are useful in the study of partial differential equations. Rochberg and Tachizawa 18 proved that certain Gabor frames almost diagonalize pseudodifferential operators whose symbols are in a class similar to that of the classical symbol classes used in partial differential equations. In [17, Gröchenig and Heil proved a similar result for pseuodifferential operators with symbol in the modulation space $M^{\infty, 1}$, also known as Sjöstrand's class.

These wavelet and Gabor frames share the property of being localized frames, which gives us a clue as to which types of frames may simultaneously almost diagonalize operators of a particular class. They also share the property of being Banach

Received by the editors January 22, 2009, and, in revised form, April 5, 2009.

2000 Mathematics Subject Classification. Primary 42C15, 46B15, 47B37, 47L80.

Key words and phrases. Localized frames, localizable operators, Banach frames.

(C)2009 American Mathematical Society Reverts to public domain 28 years from publication 
frames for a family of Banach spaces, the Besov and modulation spaces respectively. We shall prove that if an operator is almost diagonalized or "localizable" by a pair of localized frames, then it is bounded on a collection of associated Banach spaces.

In addition to boundedness, we shall show that if the operator is surjective, then we have a method of constructing new localized frames. This stems from the fact that by applying a bounded surjective linear operator to a frame, we again get a frame 11. We focus on frames generated by unitary shifts of a single atom, which include Gabor frames, wavelet frames, and shift-invariant frames.

The paper is organized as follows. Section 2 reviews basic concepts and the concept of symmetric localization of frames. Section 3 introduces the concept of localized operators and in Section 4, we investigate the algebraic structure of $\ell^{1}$ localizable operators in particular. In Section 5, we prove the boundedness of these operators on an associated family of Banach spaces. In Section 6, we introduce a method of constructing localized frames using localizable operators, and finally in Section 7, we explore some examples.

\section{BASIC CONCEPTS AND FRAME LOCALIZATION}

Here, we review some basic concepts and fix notation. We recommend [6, 14] for additional background.

Let $\mathcal{F}=\left(f_{x}\right)_{x \in X} \subset \mathcal{H}$, a separable Hilbert space, where $X$ is a countable indexing set. $\mathcal{F}$ is a frame for $\mathcal{H}$ with frame bounds $A, B>0$ if we have the following for all $f \in \mathcal{H}: A\|f\|^{2} \leq \sum_{x \in X}\left|\left\langle f, f_{x}\right\rangle\right|^{2} \leq B\|f\|^{2}$. The analysis operator $C:=C_{\mathcal{F}}: \mathcal{H} \rightarrow \ell^{2}(X), C f=\left(\left\langle f, f_{x}\right\rangle\right)_{x \in X}$ is bounded and linear on $\mathcal{H}$. The adjoint of $C$ is the synthesis operator $C^{*}: \ell^{2}(X) \rightarrow \mathcal{H}, C^{*}\left(\left(c_{x}\right)_{x \in X}\right)=\sum_{x \in X} c_{x} f_{x}$. The frame operator $S=C^{*} C: \mathcal{H} \rightarrow \mathcal{H}, S f=\sum_{x \in X}\left\langle f, f_{x}\right\rangle f_{x}$ is a bounded, positive, invertible operator on $\mathcal{H}$. The Gramian matrix $\left[\left\langle f_{x_{1}}, f_{x_{2}}\right\rangle\right]_{X X}$ representing $C C^{*}: \ell^{2}(X) \rightarrow \ell^{2}(X)$, is a bounded linear operator on $\ell^{2}(X)$. The crossGramian matrix $\left[\left\langle f_{x}, e_{y}\right\rangle\right]_{Y X}$ representing $C_{\mathcal{E}} C_{\mathcal{F}}^{*}: \ell^{2}(X) \rightarrow \ell^{2}(Y)$, compares two frames $\mathcal{F}$ and $\mathcal{E}$. A dual frame $\tilde{\mathcal{F}}=\left(\tilde{f}_{x}\right)_{x \in X}$ allows for the stable reconstruction $f=\sum_{x \in X}\left\langle f, \tilde{f}_{x}\right\rangle f_{x}$. The invertibility of the frame operator ensures the existence of at least one dual frame of $\mathcal{F}$, called the canonical dual frame, $\left(S^{-1} f_{x}\right)_{x \in X}$.

In the following, let $G$ be a group of the form $\prod_{i=1}^{d} a_{i} \mathbb{Z} \times \prod_{j=1}^{e} \mathbb{Z}_{n_{j}}$. For every $g=$ $\left(a_{1} n_{1}, a_{2} n_{2}, \ldots, a_{d} n_{d}, m_{1}, m_{2}, \ldots, m_{e}\right) \in G$, let

$$
|g|=\sup \left\{\left|a_{1} n_{1}\right|,\left|a_{2} n_{2}\right|, \ldots,\left|a_{d} n_{d}\right|, \delta\left(m_{1}\right), \delta\left(m_{2}\right), \ldots, \delta\left(m_{e}\right)\right\},
$$

where

$$
\delta\left(m_{j}\right)= \begin{cases}0 & \text { if } m_{j}=0 \\ 1 & \text { otherwise }\end{cases}
$$

Define a metric on $G$ to be $d(g, h)=|g-h|$ for $g, h \in G$.

For a subset $S \subset X$, define $|S|:=\#[S]$, the cardinality of $S$.

Definition 2.1 (Symmetric localization). Let $\mathcal{F}=\left(f_{x}\right)_{x \in X}$ and $\mathcal{E}=\left(e_{y}\right)_{y \in Y}$ be frames for a separable Hilbert space $\mathcal{H}$, where $X, Y \subset \mathbb{R}^{d}$ are countable index sets. Assume that there exist maps $a_{X}: X \rightarrow G, a_{Y}: Y \rightarrow G$ such that $\max \left\{\sup _{j \in G}\left|a_{X}^{-1}(j)\right|, \sup _{j \in G}\left|a_{Y}^{-1}(j)\right|\right\} \leq K<\infty$. 
(1) $(\mathcal{F}, \mathcal{E})$ is symmetrically $\ell^{p}$-localized if there exists an $r \in \ell^{p}(G)$ such that for all $x \in X, y \in Y$,

$$
\left|\left\langle f_{x}, e_{y}\right\rangle\right| \leq r_{a_{X}(x)-a_{Y}(y)} .
$$

(2) $\mathcal{F}$ is $\ell^{p}$-self-localized if it is symmetrically $\ell^{p}$-localized with respect to itself.

Remark 2.2. In the simplest case where $X=Y=G$ and the maps $a_{X}=a_{Y}=i d$, $(\mathcal{F}, \mathcal{E})$ is symmetrically $\ell^{p}$-localized can be understood to mean that the crossGramian matrix $\left[\left\langle f_{x}, e_{y}\right\rangle\right]_{Y X}$ has off-diagonal $\ell^{p}$-decay.

This definition, as well as all other definitions and theorems in this paper, can be modified to include $v$-moderate weights, where $v$ is a submultiplicative weight. We omit weights here for the sake of simplicity.

This idea of frame localization was introduced independently in [15, 3]. To distinguish the symmetric localization definition from their definitions, we call this "symmetric $\ell^{p}$-localization". The advantage of this definition is that the localization property is independent of the indexing of the frame elements. All of these definitions are closely related and the main results from both their papers also hold for the symmetric definition [11, 12.

Theorem 2.3 (3, 9]). Let $\mathcal{F}$ be an $\ell^{1}$-self-localized frame. Then the canonical dual frame $\tilde{\mathcal{F}}$ is also an $\ell^{1}$-self-localized frame and $(\mathcal{F}, \tilde{\mathcal{F}})$ is symmetrically $\ell^{1}$-localized.

Localized frames have the nice property of being Banach frames for an associated family of Banach spaces. We define these concepts below.

Definition 2.4. Let $B^{\prime}$ be the dual of a Banach space $B$. $\mathcal{F}=\left(f_{x}\right)_{x \in X} \subset B^{\prime}$ is called a Banach frame for $B$ if there exists a Banach space $B_{d}(X)$ of sequences associated to $B$ such that the following properties hold:

(a) The coefficient operator $C_{\mathcal{E}}: B \rightarrow B_{d}$ defined by $C_{\mathcal{E}} f=\left(f_{x}(f)\right)_{x \in X}$ is bounded.

(b) We have the norm equivalence $\|f\|_{B} \asymp\left\|f_{x}(f)\right\|_{B_{d}}$.

(c) There exists a bounded operator $R: B_{d} \rightarrow B$, called the reconstruction operator, such that $R\left(\left(f_{x}(f)\right)_{x \in X}\right)=f$ for all $f \in B$.

Definition 2.5. Let $\mathcal{E}$ be an $\ell^{1}$-self-localized frame for $\mathcal{H}$ and $\tilde{\mathcal{E}}$ its canonical dual. Let $\mathcal{H}_{0} \subseteq \mathcal{H}$ be the subspace spanned by finite linear combinations of elements in $\mathcal{E}$. For $1 \leq p<\infty$, we define the $\mathcal{H}^{p}$-norm on $\mathcal{H}_{0}$ by

$$
\|f\|_{\mathcal{H}^{p}}=\left\|C_{\tilde{\mathcal{E}}} f\right\|_{\ell^{p}}=\left\|\left\langle f, \tilde{e}_{y}\right\rangle\right\|_{\ell^{p}} .
$$

Then $\mathcal{H}^{p}(\mathcal{E}, \tilde{\mathcal{E}})$ is defined to be the $\mathcal{H}^{p}$-norm completion of $\mathcal{H}_{0}$. For $p=\infty, \mathcal{H}^{\infty}$ is the completion of $\mathcal{H}_{0}$ in the $\sigma\left(\mathcal{H}, \mathcal{H}_{0}\right)$ topology.

By definition, $\mathcal{H}^{p}$ is a Banach space and the set of all $\mathcal{H}^{p}$ such that $1 \leq p \leq \infty$ is called the family of Banach spaces associated with $\mathcal{E}$. We may also use alternate duals rather than the canonical dual; however, to make sure that all the theorems in this paper hold for alternate duals, we must assume further that the dual frame is $\ell^{1}$-localized and is $\ell^{1}$-localized with respect to $\mathcal{E}$. 


\section{LOCALIZABLE OPERATORS}

3.1. Definition of localizable operators. We intuitively understand localized frames as having Gramian matrices with off-diagonal decay. We introduce the notion of localizable operators, which we intuitively understand as having a matrix representation with off-diagonal decay.

Definition 3.1. Let $\mathcal{T} \in \mathcal{L}(\mathcal{H})$, the set of bounded linear operators on a separable Hilbert space. Let $\mathcal{F}=\left(f_{x}\right)_{x \in X}, \mathcal{E}=\left(e_{y}\right)_{y \in Y}$ be frames for $\mathcal{H}$ and let $\tilde{\mathcal{E}}$ be a dual frame of $\mathcal{E}$. Let $a_{X}: X \rightarrow G, a_{Y}: Y \rightarrow G$ be maps such that for all $j \in G$, $\max \left\{\sup _{j \in G}\left|a_{X}^{-1}(j)\right|, \sup _{j \in G}\left|a_{Y}^{-1}(j)\right|\right\} \leq K<\infty$. Then $\mathcal{T}$ is an $\ell^{p}$-localizable operator with respect to $(\mathcal{F}, \tilde{\mathcal{E}})$ if there exists an $r \in \ell^{p}(G)$ such that for all $x \in X$ and $y \in Y$,

$$
\left|\left\langle\mathcal{T} f_{x}, \tilde{e}_{y}\right\rangle\right| \leq r_{a_{X}(x)-a_{Y}(y)} .
$$

Notice that $(\mathcal{F}, \mathcal{E})$ is symmetrically $\ell^{p}$-localized if and only if the frame operator $S$ of $\mathcal{E}$ is $\ell^{p}$-localizable with respect to $(\mathcal{F}, \tilde{\mathcal{E}})$.

Let $A=\left[\left\langle\mathcal{T} f_{x}, \tilde{e}_{y}\right\rangle\right]_{Y X}$, which we can think of as a matrix representation of $\mathcal{T}$. With regard to boundedness, $\mathcal{T}$ is bounded on $\mathcal{H}$ if and only if $A$ is bounded on $\ell^{2}(X)$. This allows us to work at the discrete level with $A$ when determining boundedness. This result is an immediate corollary of the proposition below.

Proposition 3.2. Let $\mathcal{F}=\left(f_{x}\right)_{x \in X}$ and $\mathcal{E}=\left(e_{y}\right)_{y \in Y}$ be frames for $\mathcal{H}$, with dual frames $\tilde{\mathcal{F}}$ and $\tilde{\mathcal{E}}$. Then the following diagram commutes:

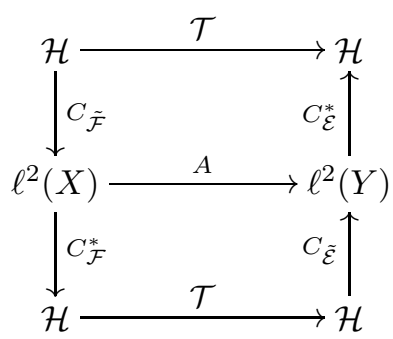

Proof. Let $f \in \mathcal{H}$ and $c \in \ell^{2}(X)$. Then

$$
\begin{aligned}
\mathcal{T} f & =\sum_{x \in X}\left\langle f, \tilde{f}_{x}\right\rangle \mathcal{T} f_{x}=\sum_{y \in Y} \sum_{x \in X}\left\langle f, \tilde{f}_{x}\right\rangle\left\langle\mathcal{T} f_{x}, \tilde{e}_{y}\right\rangle e_{y} \\
& =C_{\mathcal{E}}^{*}\left(\left\{\sum_{x \in X}\left\langle f, \tilde{f}_{x}\right\rangle\left\langle\mathcal{T} f_{x}, \tilde{e}_{y}\right\rangle\right\}_{y \in Y}\right)=C_{\mathcal{E}}^{*} A C_{\tilde{\mathcal{F}}} f .
\end{aligned}
$$

Thus,

$$
A c=\left\{\sum_{x \in X} c_{x}\left\langle\mathcal{T} f_{x}, \tilde{e}_{y}\right\rangle\right\}_{y \in Y}=C_{\tilde{\mathcal{E}}}\left(\sum_{x \in X} c_{x} \mathcal{T} f_{x}\right)=C_{\tilde{\mathcal{E}}}\left(\mathcal{T} \sum_{x \in X} c_{x} f_{x}\right)=C_{\tilde{\mathcal{E}}} \mathcal{T} C_{\mathcal{F}}^{*} c .
$$

Notice that this result holds for any dual frames $\tilde{\mathcal{F}}$ and $\tilde{\mathcal{E}}$, as the analysis operators of the dual frames are linear and bounded. In the following, let $\tilde{\mathcal{E}}$ denote a dual frame for $\mathcal{E}$ which may or may not be the canonical dual. 
Theorem 3.3. Let $\mathcal{E}=\left(e_{y}\right)_{y \in Y}, \mathcal{F}=\left(f_{x}\right)_{x \in X}, \mathcal{G}=\left(g_{z}\right)_{z \in Z}$ be frames for $\mathcal{H}$ and $\mathcal{T}, \mathcal{S} \in \mathcal{L}(\mathcal{H})$. If $\mathcal{T}$ is $\ell^{1}$-localizable with respect to $(\mathcal{F}, \tilde{\mathcal{E}})$, and if $\mathcal{S}$ is $\ell^{1}$-localizable with respect to $(\mathcal{E}, \tilde{\mathcal{G}})$, then $\mathcal{S} \mathcal{T}$ is $\ell^{1}$-localizable with respect to $(\mathcal{F}, \tilde{\mathcal{G}})$.

Proof. Let $r, s \in \ell^{1}(G)$ be such that $\left|\left\langle\mathcal{T} f_{x}, \tilde{e}_{y}\right\rangle\right| \leq r_{a_{X}(x)-a_{Y}(y)}$ and $\left|\left\langle\mathcal{S} e_{y}, \tilde{g}_{z}\right\rangle\right| \leq$ $s_{a_{Y}(y)-a_{Z}(z)}$. Define $t:=r * s \in \ell^{1}(G)$. Then

$$
\begin{aligned}
\left|\left\langle\mathcal{S T} f_{x}, \tilde{g}_{z}\right\rangle\right| & \leq \sum_{y \in Y}\left|\left\langle\mathcal{T} f_{x}, \tilde{e}_{y}\right\rangle\right|\left|\left\langle\mathcal{S} e_{y}, \tilde{g}_{z}\right\rangle\right| \\
& \leq \sum_{y \in Y} r_{a_{X}(x)-a_{Y}(y)} s_{a_{Y}(y)-a_{Z}(z)} \\
& \leq K \sum_{g \in G} r_{a_{X}(x)-g} s_{g-a_{Z}(y)} \\
& \leq K(r * s)_{a_{X}(x)-a_{Z}(z)}=K t_{a_{X}(x)-a_{Z}(z)} .
\end{aligned}
$$

\section{Algebra of $\ell^{1}$-LOCAlizable operators}

We introduce a Banach algebra of operators $\mathcal{T} \in \mathcal{L}(\mathcal{H})$. In the process, we expand the idea of a Banach algebra of infinite matrices proposed in [3]. We first establish that the localizability of $\mathcal{T}$ is not dependent on the particular pair of frames. Assume for the remainder of this paper that for a frame $\mathcal{E}, \tilde{\mathcal{E}}$ refers to the canonical dual frame of $\mathcal{E}$.

Theorem 4.1. Let $\mathcal{F}, \mathcal{F}^{\prime}, \mathcal{E}$ and $\mathcal{E}^{\prime}$ be $\ell^{1}$-self-localized frames. Suppose $\left(\mathcal{F}^{\prime}, \mathcal{F}\right)$ and $\left(\mathcal{E}^{\prime}, \mathcal{E}\right)$ are symmetrically $\ell^{1}$-localized. If $\mathcal{T}$ is $\ell^{1}$-localizable with respect to $(\mathcal{F}, \tilde{\mathcal{E}})$, then $\mathcal{T}$ is $\ell^{1}$-localizable with respect to $\left(\mathcal{F}^{\prime}, \tilde{\mathcal{E}}^{\prime}\right)$.

Proof. This follows from Theorem 3.3 and Theorem 2.3 .

Thus, we can make the following definition.

Definition 4.2. Let $\mathcal{T} \in \mathcal{L}(\mathcal{H})$ and let $\mathcal{F}=\left(f_{x}\right)_{x \in X}, \mathcal{E}=\left(e_{x}\right)_{x \in X}$ be $\ell^{1}$-selflocalized frames for $\mathcal{H}$. We say that $\mathcal{T} \in \mathcal{L}(\mathcal{H})^{1}$ if $\mathcal{T}$ is $\ell^{1}$-localizable with respect to $(\mathcal{F}, \tilde{\mathcal{E}})$.

Theorem 4.3. Let $\mathcal{L}(\mathcal{H})^{1} \subset \mathcal{L}(\mathcal{H})$ be endowed with the same norm as $\mathcal{L}(\mathcal{H})$. The following statements hold:

(1) The identity I belongs to $\mathcal{L}(\mathcal{H})^{1}$.

(2) $\mathcal{L}(\mathcal{H})^{1}$ is closed under addition and composition.

(3) $\mathcal{L}(\mathcal{H})^{1}$ is complete with respect to the operator norm.

In particular, $\mathcal{L}(\mathcal{H})^{1}$ is a unital Banach sub-algebra of $\mathcal{L}(\mathcal{H})$.

Proof. (1) This follows from the fact that $I$ is always $\ell^{1}$-localized with respect to $\ell^{1}$-localized frames.

(2) It is clear that $\mathcal{L}(\mathcal{H})^{1}$ is closed under addition, since for $r, s \in \ell^{1}(G), r+s \in$ $\ell^{1}(G)$. The property of being closed under composition follows from Theorem 3.3 .

(3) Consider the Cauchy sequence $\left\{\mathcal{T}_{n}\right\}_{n \in \mathbb{N}}$ of operators in $\mathcal{L}(\mathcal{H})^{1}$. Certainly, this Cauchy sequence converges to some $\mathcal{T} \in \mathcal{L}(\mathcal{H})$, and for every fixed $x$ and every fixed $y,\left\langle\mathcal{T}_{n} f_{x}, \tilde{e}_{y}\right\rangle$ converges to $\left\langle\mathcal{T} f_{x}, \tilde{e}_{y}\right\rangle$. We need only to prove that $\left|\left\langle\mathcal{T} f_{x}, \tilde{e}_{y}\right\rangle\right| \leq$ $r_{a_{X}(x)-a_{Y}(y)}$ for some $r \in \ell^{1}(G)$. 
Notice that for each $\mathcal{T}_{n}$, there is an $r^{n} \in \ell^{1}(G)$ such that

$$
\left|\left\langle\mathcal{T}_{n} f_{x}, \tilde{e}_{y}\right\rangle\right| \leq r_{a_{X}(x)-a_{Y}(y)}^{n} .
$$

Let $\epsilon>0$. Then there is an $N_{\epsilon} \in \mathbb{N}$ such that for all $n, m \geq N_{\epsilon},\left\|T_{n}-T_{m}\right\|<\epsilon$ and we can define $r^{n, m} \in \ell^{1}(G)$ so that

$$
\left|\left\langle\left(\mathcal{T}_{n}-\mathcal{T}_{m}\right) f_{x}, \tilde{e}_{y}\right\rangle\right| \leq r_{a_{X}(x)-a_{Y}(y)}^{n, m}, \quad\left\|r^{n, m}\right\|<\epsilon .
$$

Consider $\epsilon_{k}=\frac{1}{2^{k}}$ for $k \in \mathbb{N}$, and let $N_{k}=N_{\epsilon_{k}}$ as above. For $k=0$, let $N_{0}=0$ and $T_{0}=0$. Define $r=r^{N_{1}}+\sum_{k=1}^{\infty} r^{N_{k+1}, N_{k}}$. Notice that

$$
\|r\| \leq\left\|r^{N_{1}}\right\|+\sum_{k=1}^{\infty}\left\|r^{N_{k+1}, N_{k}}\right\|<\left\|r^{N_{1}}\right\|+\sum_{k=1}^{\infty} \frac{1}{2^{k}}<\infty .
$$

So $r \in \ell^{1}(G)$ and

$$
\begin{aligned}
\left|\left\langle\mathcal{T} f_{x}, \tilde{e}_{y}\right\rangle\right| & =\lim _{k \rightarrow \infty}\left|\left\langle\mathcal{T}_{N_{k}} f_{x}, \tilde{e}_{y}\right\rangle\right| \\
& \leq \lim _{n \rightarrow \infty} \sum_{k=0}^{n}\left(\left|\left\langle\mathcal{T}_{N_{k+1}} f_{x}, \tilde{e}_{y}\right\rangle\right|-\left|\left\langle\mathcal{T}_{N_{k}} f_{x}, \tilde{e}_{y}\right\rangle\right|\right) \\
& \leq \lim _{n \rightarrow \infty} \sum_{k=0}^{n}\left|\left\langle\left(\mathcal{T}_{N_{k+1}}-\mathcal{T}_{N_{k}}\right) f_{x}, \tilde{e}_{y}\right\rangle\right| \\
& <r_{a_{X}(x)-a_{Y}(y)}^{N_{1}}+\sum_{k=1}^{\infty} r_{a_{X}(x)-a_{Y}(y)}^{N_{k+1}, N_{k}}=r_{a_{X}(x)-a_{Y}(y)} .
\end{aligned}
$$

Hence, $\mathcal{T} \in \mathcal{L}(\mathcal{H})^{1}$.

Theorem 4.4. If $\mathcal{T} \in \mathcal{L}(\mathcal{H})^{1}$ is invertible, then $\mathcal{T}^{-1} \in \mathcal{L}(\mathcal{H})^{1}$.

Proof. Suppose $\mathcal{T} \in \mathcal{L}(\mathcal{H})^{1}$, and assume that there exist $\ell^{1}$-self-localized frames $\mathcal{F}=\left(f_{x}\right)_{x \in X}, \mathcal{E}=\left\{e_{y}\right\}_{y \in X}$ such that $\left|\left\langle\mathcal{T} f_{x}, \tilde{e}_{y}\right\rangle\right| \leq r_{a_{X}(x)-a_{X}(y)}, r \in \ell^{1}(G)$. Assume the frame bounds for $\mathcal{F}$ and $\mathcal{E}$ are $\alpha, \beta>0$ and $\alpha^{\prime}, \beta^{\prime}>0$ respectively. The associated matrix representation is $A=\left[\left\langle\mathcal{T} f_{x}, \tilde{e}_{y}\right\rangle\right]_{x, y \in X}$. Consider the matrix $A^{\dagger}=\left[\left\langle\mathcal{T}^{-1} e_{y}, \tilde{f}_{x}\right\rangle\right]_{x, y \in X}$. By Theorem 3.2 , $A^{\dagger}$ can be represented by the operator $C_{\tilde{\mathcal{F}}} \mathcal{T}^{-1} C_{\mathcal{E}}^{*}$. Likewise, $A$ can be represented by the operator $C_{\tilde{\mathcal{E}}} \mathcal{T} C_{\mathcal{F}}^{*}$. It is clear that $A A^{\dagger}$ and $A^{\dagger} A$ are the orthogonal projections onto the ranges of $A$ and $A^{\dagger}$ respectively. Hence, we have that $A^{\dagger}$ is the pseudo-inverse of $A$.

We can now use similar proof techniques as found in $[3$, which in their case proved that the canonical dual of an $\ell^{1}$-self-localized frame is $\ell^{1}$-self-localized. It suffices to consider the case where the index set $X=G . A$ is a bounded operator in the set $\mathcal{L}\left(\ell^{2}(G)\right)$, with spectrum $\operatorname{Sp}_{\mathcal{L}\left(\ell^{2}(G)\right)}(A)$ being a closed set in $\{0\} \cup\left[\left(\alpha / \beta^{\prime}\right)^{1 / 2}\|T\|,\left(\beta / \alpha^{\prime}\right)^{1 / 2}\|T\|\right] . A$ is also a bounded operator in the algebra $\mathcal{B}(G)^{1}$ of matrices with off-diagonal $\ell^{1}$-decay, as defined in [3], and $\operatorname{Sp}_{\mathcal{L}\left(\ell^{2}(G)\right)}(A)=$ $\mathrm{Sp}_{\mathcal{B}(G)^{1}}(A)$.

Standard holomorphic calculus and a theorem proved by Gohberg, Kaashoek and Woerdeman [13, Baskakov [5], and Sjöstrand [19] give us that the pseudo-inverse $A^{\dagger}$ is also an element of $B(G)^{1}$, hence satisfying the conditions for $\mathcal{T}^{-1} \in \mathcal{L}(\mathcal{H})^{1}$. 


\section{BOUNDEDNESS ON BANACH SPACES}

If $\mathcal{T} \in \mathcal{L}(\mathcal{H})$ is localized with respect to localized frames $(\mathcal{F}, \mathcal{E})$, with the matrix representation $\left[\left\langle\mathcal{T} f_{x}, e_{y}\right\rangle\right]_{X Y}$ having the same type of decay as the cross-Gramian matrix, then we shall prove below that the operator can be extended as a bounded operator on the associated family of Banach spaces $\mathcal{H}^{p}$ (Definition 2.5). As an example, Gabor frames with nice generator functions are examples of localized frames, and as we have mentioned, they almost diagonalize classes of pseudodifferential operators. One property that we need is that localized (Hilbert) frames are also Banach frames for some associated family of Banach spaces as described in [9, 11. Specifically, we have the following.

Theorem 5.1 (11]). Suppose $\mathcal{F}=\left(f_{x}\right)_{x \in X}$ is a frame and $\mathcal{E}=\left(e_{y}\right)_{y \in Y}$ an $\ell^{1}$ self-localized Riesz basis for $\mathcal{H}$. If $(\mathcal{F}, \mathcal{E})$ is symmetrically $\ell^{1}$-localized, then $\mathcal{F}$ is a Banach frame for the family of Banach spaces $\mathcal{H}^{p}(\mathcal{E}, \tilde{\mathcal{E}})$.

Theorem 5.2. Assume $\mathcal{E}=\left(e_{y}\right)_{y \in Y}$ is an $\ell^{1}$-self-localized Riesz basis and assume $\mathcal{F}=\left(f_{x}\right)_{x \in X}$ is a frame with dual frame $\tilde{\mathcal{F}}$ such that $(\tilde{\mathcal{F}}, \mathcal{E})$ is symmetrically $\ell^{1}$ localized. Suppose the following conditions are satisfied:

- $\mathcal{T} \in \mathcal{L}(\mathcal{H})$

- $\mathcal{T}$ is $\ell^{1}$-localizable with respect to $(\mathcal{F}, \widetilde{\mathcal{E}})$.

Then $\mathcal{T}$ is bounded on $\mathcal{H}^{p}$ for all $1 \leq p \leq \infty$.

Proof. Let $\mathcal{H}_{0}$ be as in Definition 2.5. Then $\mathcal{H}_{0}$ is dense in $\mathcal{H}^{p}$. So it suffices to prove $\|T f\|_{\mathcal{H}^{p}} \leq C\|f\|_{\mathcal{H}^{p}}$ for all $f \in \mathcal{H}_{0}$, where $C$ does not depend on $f$. Let $f \in \mathcal{H}_{0}$, $f=\sum_{y \in Y} c_{y} e_{y}$, where all but finitely many $c_{y}$ are nonzero. By Theorem 5.1, $\tilde{\mathcal{E}}$ is a Banach frame for $\mathcal{H}^{p}$, so the reconstruction operator $R: \ell^{p}(Y) \rightarrow \mathcal{H}^{p}$ defined by $R c=\sum_{y \in Y} c_{y} e_{y}$ is bounded. Thus we have the following:

$$
\begin{aligned}
\|\mathcal{T} f\|_{\mathcal{H}^{p}} & =\left\|\sum_{y \in Y} c_{y} \mathcal{T} e_{y}\right\|_{\mathcal{H}^{p}} \\
& =\left\|\sum_{y \in Y} c_{y} \sum_{x \in X} \sum_{z \in Y}\left\langle e_{y}, \tilde{f}_{x}\right\rangle\left\langle\mathcal{T} f_{x}, \tilde{e}_{z}\right\rangle e_{z}\right\|_{\mathcal{H}^{p}} \\
& \leq k\left\|\left(\sum_{y \in Y} c_{y} \sum_{x \in X}\left\langle e_{y}, \tilde{f}_{x}\right\rangle\left\langle\mathcal{T} f_{x}, \tilde{e}_{z}\right\rangle\right)_{z \in Y}\right\|_{l^{p}(Y)} .
\end{aligned}
$$

Define $d \in l^{p}(G)$ by

$$
d_{j}= \begin{cases}\sum_{y \in a_{Y}^{-1}(j)} c_{y} & \text { if } a_{Y}^{-1}(j) \neq \emptyset \\ 0 & \text { if } a_{Y}^{-1}(j)=\emptyset .\end{cases}
$$

As shown in [12, since $\max \left\{\sup _{j \in G}\left|a_{X}^{-1}(j)\right|, \sup _{j \in G}\left|a_{Y}^{-1}(j)\right|\right\} \leq K$, we have that $\|d\|_{l^{p}(G)} \leq K\|c\|_{l^{p}(Y)}$. By this and our assumptions, we have the following:

$$
\begin{aligned}
\|\mathcal{T} f\|_{\mathcal{H}^{p}} & \leq k\left\|\left(\sum_{y \in Y} c_{y} \sum_{x \in X} r_{a_{Y}(y)-a_{X}(x)} s_{a_{X}(x)-a_{Y}(z)}\right)_{z \in Y}\right\|_{l^{p}(Y)} \\
& \leq k\left\|\left(\sum_{y \in Y} c_{y} \sum_{j \in G} \sum_{x \in a_{X}^{-1}(j)} r_{a_{Y}(y)-a_{X}(x)} s_{a_{X}(x)-a_{Y}(z)}\right)_{z \in Y}\right\|_{l^{p}(Y)}
\end{aligned}
$$




$$
\begin{aligned}
& \leq k K\left\|\left(\sum_{y \in Y} c_{y}(r * s)_{a_{Y}(y)-a_{Y}(z)}\right)_{z \in Y}\right\|_{l^{p}(Y)} \\
& =k K\left\|\left(\sum_{j \in G} d_{j}(r * s)_{j-a_{Y}(z)}\right)_{z \in Y}\right\|_{l^{p}(Y)} \\
& =k K\left\|\left((d * r * s)_{a_{Y}(z)}\right)_{z \in Y}\right\|_{l^{p}(Y)} \\
& \leq k K^{2}\left\|\left((d * r * s)_{j}\right)_{j \in G}\right\|_{l^{p}(G)} \\
& \leq k K^{2}\|d\|_{l^{p}(G)}\|r * s\|_{l^{1}(G)} \\
& \leq k K^{3}\|r * s\|_{l^{1}(G)}\|c\|_{l^{p}(Y)} \\
& =k K^{3}\|r * s\|_{l^{1}(G)}\|f\|_{\mathcal{H}^{p}} .
\end{aligned}
$$

Corollary 5.3. Let $\mathcal{E}$ be an $\ell^{1}$-self-localized Riesz basis and let $\mathcal{T} \in \mathcal{L}(\mathcal{H})$. If $\mathcal{T}$ is $\ell^{1}$-localizable with respect to $(\mathcal{E}, \widetilde{\mathcal{E}})$, then $\mathcal{T}$ is bounded on $\mathcal{H}^{p}$ for all $1 \leq p \leq \infty$.

Remark 5.4. If $\mathcal{F}$ is an $\ell^{1}$-self-localized frame, then Theorem 5.2 is true if the symmetric $\ell^{1}$-localization of $(\widetilde{\mathcal{F}}, \mathcal{E})$ is replaced by $(\mathcal{F}, \mathcal{E})$.

\section{The CONSTRUCTION OF LOCALIZED FRAMES}

This technique of looking at matrix representations with respect to frames helped us to extend localizable operators bounded on $\mathcal{H}$ to a family of Banach spaces. In this section, we shall see that this technique also leads us to a new way of constructing localized frames. In the process, we will identify new classes of operators localized with respect to frames constructed from unitary shifts of a single atom function. We have the following theorem concerning the role of operators in determining a relationship between frames.

Theorem 6.1 ([1]). Let $\mathcal{F}=\left(f_{x}\right)_{x \in X}$ be a frame for $\mathcal{H}$. If $\mathcal{T}$ is a bounded linear operator on $\mathcal{H}$, then $\left(\mathcal{T} f_{x}\right)_{x \in X}$ is a frame for $\mathcal{H}$ if and only if there exists a positive constant $\gamma$ such that for every $f \in \mathcal{H}$,

$$
\left\|\mathcal{T}^{*} f\right\|^{2} \geq \gamma\|f\|^{2}
$$

Suppose $\mathcal{F}$ is an $\ell^{1}$-self-localized frame. We address the question, what are the conditions on an operator $T$ such that $\left(\mathcal{T} f_{x}\right)_{x \in X}$ is $\ell^{1}$-self-localized? It is only known that the canonical dual of an $\ell^{1}$-self-localized frame, $\left(S^{-1} f_{x}\right)_{x \in X}$, is again an $\ell^{1}$-self-localized frame. Although (6.1) guarantees that $\left(\mathcal{T} f_{x}\right)_{x \in X}$ is a frame, it does not guarantee that it is a self-localized frame nor does it guarantee that this new frame extends to a Banach frame for an associated family of Banach frames.

6.1. Necessary and sufficient conditions for $\mathcal{T F}$ to be a localized frame. We give a necessary and sufficient condition for localization.

Theorem 6.2. Let $\mathcal{F}=\left(f_{x}\right)_{x \in X}$ be an $\ell^{1}$-self-localized frame, $\mathcal{T}$ a bounded linear operator which satisfies $(6.1)$. $\left(\mathcal{T} f_{x}\right)_{x \in X}$ is an $\ell^{1}$-self-localized frame if and only if $\mathcal{T}^{*} \mathcal{T}$ is $\ell^{1}$-localizable with respect to $(\mathcal{F}, \tilde{\mathcal{F}})$.

Proof. Suppose $\left(\mathcal{T} f_{x}\right)_{x \in X}$ is an $\ell^{1}$-self-localized frame. We have that $\left\langle\mathcal{T}^{*} \mathcal{T} f_{x}, f_{y}\right\rangle=$ $\left\langle\mathcal{T} f_{x}, \mathcal{T} f_{y}\right\rangle$. But $\left\{\mathcal{T} f_{x}\right\}_{x \in X}$ being an $\ell^{1}$-self-localized frame is equivalent to the 
identity operator $I$ on $\mathcal{H}$ being $\ell^{1}$-localizable with respect to $(\mathcal{F}, \mathcal{F})$. But by Theorem $2.3,(\mathcal{F}, \tilde{\mathcal{F}})$ is symmetrically $\ell^{1}$-localized. Hence, equivalently, the identity operator $I$ is $\ell^{1}$-localizable with respect to $(\mathcal{F}, \tilde{\mathcal{F}})$. It then follows by Theorem 3.3 that $\mathcal{T}^{*} \mathcal{T}$ is $\ell^{1}$-localizable with respect to $(\mathcal{F}, \tilde{\mathcal{F}})$ since it is $\ell^{1}$-localizable with respect to $(\mathcal{F}, \mathcal{F}), I$ is $\ell^{1}$-localizable with respect to $(\mathcal{F}, \tilde{\mathcal{F}})$, and $\mathcal{T}^{*} \mathcal{T}=I \mathcal{T}^{*} \mathcal{T}$.

Suppose $\mathcal{T}^{*} \mathcal{T}$ is $\ell^{1}$-localizable with respect to $(\mathcal{F}, \tilde{\mathcal{F}})$. Then $\mathcal{T}^{*} \mathcal{T}=I \mathcal{T}^{*} \mathcal{T}$ is $\ell^{1}$ localizable with respect to $(\mathcal{F}, \mathcal{F})$. But we have $\left\langle\mathcal{T}^{*} \mathcal{T} f_{x}, f_{y}\right\rangle=\left\langle\mathcal{T} f_{x}, \mathcal{T} f_{y}\right\rangle$. Thus $\left\{\mathcal{T} f_{x}\right\}_{x \in X}$ is an $\ell^{1}$-self-localized frame.

Notice that if $\mathcal{T}$ is a unitary operator, the theorem is automatically satisfied. If we only want to ask the question of whether or not $\mathcal{T} \mathcal{F}$ is an $\ell^{1}$-localized frame given that $\mathcal{F}$ is an $\ell^{1}$-self-localized frame, we have the following sufficient condition.

Theorem 6.3. Let $\mathcal{F}=\left(f_{x}\right)_{x \in X}$ be an $\ell^{1}$-self-localized frame, $\mathcal{T}$ a bounded linear operator which satisfies $(6.1)$. If $\mathcal{T}$ is $\ell^{1}$-localizable with respect to $(\mathcal{F}, \tilde{\mathcal{F}})$, then $\left(\mathcal{T} f_{x}\right)_{x \in X}$ is an $\ell^{1}$-self-localized frame.

Proof. $\left(\mathcal{T} f_{x}\right)_{x \in X}$ is a frame. Since $\mathcal{F}=\left(f_{x}\right)_{x \in X}$ is an $\ell^{1}$-self-localized frame, the identity operator $I$ is $\ell^{1}$-localizable with respect to $(\mathcal{F}, \mathcal{F}),(\tilde{\mathcal{F}}, \tilde{\mathcal{F}}),(\mathcal{F}, \tilde{\mathcal{F}})$, and $(\tilde{\mathcal{F}}, \mathcal{F})$. It follows by Theorem 3.3 that $\mathcal{T}=I \mathcal{T}$ is $\ell^{1}$-localizable with respect to $(\mathcal{F}, \mathcal{F})$ and $\mathcal{T}=\mathcal{T} I$ is $\ell^{1}$-localizable with respect to $(\tilde{\mathcal{F}}, \mathcal{F})$. But now $\mathcal{T}^{*}$ is $\ell^{1}$-localizable with respect to $(\mathcal{F}, \tilde{\mathcal{F}})$ since $\mathcal{T}=I \mathcal{T}$ is $\ell^{1}$-localizable with respect to $(\tilde{\mathcal{F}}, \mathcal{F})$. It follows that $\mathcal{T}^{*} \mathcal{T}$ is $\ell^{1}$-localizable with respect to $(\mathcal{F}, \mathcal{F})$. Using the identity operator and Theorem 3.3 again we get that $\mathcal{T}^{*} \mathcal{T}$ is $\ell^{1}$-localizable with respect to $(\mathcal{F}, \tilde{\mathcal{F}})$. Thus by Theorem $6.2\left(\mathcal{T} f_{x}\right)_{x \in X}$ is an $\ell^{1}$-self-localized frame.

\section{EXAMPLES}

7.1. Almost shift-invariant operators. Consider the shift-invariant space $V^{2}(\phi)$, where $\phi \in W\left(L^{1}\right)$, the Wiener space of locally $L^{\infty}$, globally $L^{1}$ functions. Assume it is generated by the Riesz basis $(\phi(\cdot-k))_{k \in \mathbb{Z}}=\left(T_{k} \phi\right)_{k \in \mathbb{Z}}$. It is known that $\left(T_{k} \phi\right)_{k \in \mathbb{Z}}$ is an $\ell^{1}$-self-localized basis which extends to an unconditional basis for $V^{p}(\phi), 1 \leq p \leq \infty$ [2].

We define a collection of operators which extend to bounded operators on $V^{p}(\phi)$.

Definition 7.1. Let $\left(T_{k} \phi\right)_{k \in \mathbb{Z}}$ be a Riesz basis for $V^{2}(\phi)$. Define the linear operator $\mathcal{T}$ so that $\mathcal{T} \phi \in V^{2}(\phi) \cap W\left(L^{1}\right)$ and $\mathcal{T} T_{k} \phi=a_{k} T_{k} \mathcal{T} \phi$ for some $a_{k} \in \mathbb{C}$, where the sequence $\left(a_{k}\right)_{k \in \mathbb{Z}}$ has the property that for each $k, 0<K \leq\left|a_{k}\right| \leq M<\infty$. Call all such operators almost shift-invariant.

Theorem 7.2. Let $\mathcal{T}$ be an invertible almost shift-invariant operator satisfying (1). If $\mathcal{T} \phi \in W\left(L^{1}\right)$, then for $1 \leq p \leq \infty, \mathcal{T}$ is bounded on $V^{p}(\phi)$ and $\left(\mathcal{T} T_{k} \phi\right)_{k \in \mathbb{Z}}$ is an $\ell^{1}$-localized frame which extends to a Banach frame for $V^{p}(\phi)$.

7.2. Pseudodifferential operators. Rochberg and Tachizawa [18 and Gröchenig 16 showed the boundedness of pseudodifferential operators on generalized Sobolev spaces and modulation spaces respectively using this particular technique of looking at the matrix representation with respect to Gabor frames. We focus on the results of Gröchenig.

Let $\mathcal{H}=L^{2}\left(\mathbb{R}^{d}\right)$. The pseudodifferential operator known as the Weyl transform of a symbol $\sigma(z, \zeta)$ is defined as $\sigma^{w} f(x)=\int_{\mathbb{R}^{d}} \sigma\left(\frac{x+y}{2}, \xi\right) e^{2 \pi i(x-y) \cdot \xi} f(y) d y d \xi$. Assume $\sigma(z, \zeta) \in \mathcal{S}^{\prime}\left(\mathbb{R}^{2 d}\right)$ and belongs to the Sjöstrand class. 
Theorem $7.3([16])$. The Weyl transform $\sigma^{w}$ is an $\ell^{1}$-localizable operator with respect to tight Gabor frames with atom function $g$ such that the short time Fourier transform $V_{g} g$ is in the weighted amalgam space $W\left(\ell^{1}\right)$.

Gabor frames with atom functions in $M^{\infty}$ are $\ell^{1}$-self-localized and localized with respect to each other [3, 9]. Furthermore, they are Banach frames for the associated family of modulation spaces $M^{p}[9$. Then by Theorem 5.2, we have the following previously known result (see, for example, [17]) arising from this more general context.

Theorem 7.4. Let $L_{\sigma}$ be a pseudodifferential operator such that $L_{\sigma}$ is $\ell^{1}$-localizable with respect to a pair of $\ell^{1}$-self-localized Gabor frames which are Banach frames for the modulation spaces $M^{p}, 1 \leq p \leq \infty$. Then $L_{\sigma}$ is bounded on $M^{p}, 1 \leq p \leq \infty$.

\section{ACKNOWLEDGMENTS}

The author would like to thank Akram Aldroubi and Chris Heil for their useful suggestions and comments.

\section{REFERENCES}

1. A. Aldroubi, Portraits of frames. Proc. Amer. Math. Soc. 123 (1995), 1661-1668. MR.1242070 (95g:46037)

2. A. Aldroubi and K. Gröchenig, Nonuniform sampling and reconstruction in shift-invariant spaces. SIAM Rev. 43 (2001), no. 4, 585-620. MR1882684(2003e:94040)

3. R. Balan, P.G. Casazza, C. Heil, and Z. Landau, Density, overcompleteness, and localization of frames, I. Theory, J. Fourier Anal. Appl. 12 (2006), no. 2, 105-143. MR.2224392 (2007b:42041)

4. R. Balan, P.G. Casazza, C. Heil, and Z. Landau, Density, overcompleteness, and localization of frames, II. Gabor systems, J. Fourier Anal. Appl. 12 (2006), no. 3, 309-344. MR2235170 (2007b:42042)

5. A.G. Baskakov, Wiener's theorem and asymptotic estimates for elements of inverse matrices (Russian), Funktsional. Anal. i Prilozhen. 24 (1990), no. 3, 64-65; translation in Funct. Anal. Appl. 24 (1990), no. 3, 222-224 (1991). MR.1082033 (92g:47049)

6. O. Christensen. An Introduction to Frames and Riesz Bases, Birkhäuser, Boston, 2003. MR 1946982 (2003k:42001)

7. A. Cichocki. Adaptive Blind Signal and Image Processing: Learning Algorithms and Applications, John Wiley and Sons, NJ, 2002.

8. R. Coifman and Y. Meyer, Wavelets. Calderón-Zygmund and multilinear operators, Cambridge Studies in Advanced Mathematics, 48, Cambridge Univ. Press, Cambridge, 1997. MR:1456993 (98e:42001)

9. M. Fornasier and K. Gröchenig, Intrinsic localization of frames, Constr. Approx. 22 (2005), no. 3, 395-415. MR2164142 (2006f:42030)

10. M. Frazier, B. Jawerth, and G. Weiss, Littlewood-Paley theory and the study of function spaces, CBMS Regional Conference Series in Mathematics, 79. Published for the Conference Board of the Mathematical Sciences, Washington, DC, by the American Mathematical Society, Providence, RI, 1991. MR,1107300 (92m:42021)

11. F. Futamura, Banach framed, decay in the context of localization, Sampl. Theory Signal Image Process. 6 (2007), no. 2 , 151-166. MR2343403(2008i:42062)

12. F. Futamura, Symmetrically localized frames and the removal of subsets of positive density, J. Math. Anal. Appl. 326 (2007), no. 2, 1225-1235. MR2280976 (2007k:42093)

13. I. Gohberg, M.A. Kaashoek, and H.J. Woerdeman, The band method for positive and contractive extension problems, J. Operator Theory 22 (1989), 109-155. MR.1026078 (91a:47021)

14. K. Gröchenig, Foundations of Time-Frequency Analysis, Birkhäuser, Boston, 2001. MR:1843717 (2002h:42001)

15. K. Gröchenig, Localization of frames, Banach frames, and the invertibility of the frame operator, J. Fourier Anal. Appl. 10 (2004), no. 2, 105-132. MR2054304(2005f:42086) 
16. K. Gröchenig, Time-frequency analysis of Sjöstrand's class, Rev. Mat. Iberoamericana 22 (2006), no. 2, 703-724. MR2294795 (2008b:35308)

17. K. Gröchenig and C. Heil, Modulation spaces as symbol classes for pseudodifferential operators, in: "Wavelets and Their Applications" (Chennai, January 2002), M. Krishna, R. Radha and S. Thangavelu, eds., Allied Publishers, New Delhi, 2003, 151-169.

18. R. Rochberg and K. Tachizawa, Pseudodifferential operators, Gabor frames, and local trigonometric bases, in: "Gabor Analysis and Algorithms: Theory and Applications", H.G. Feichtinger and T. Strohmer, eds., Birkhäuser, Boston, 1998, 171-192. MR.1601103 (98k:42046)

19. J. Sjöstrand, Wiener type algebras of pseudodifferential operators, Séminaire sur les Équations aux Dérivées Partielles, 1994-1995, Exp. No. IV, École Polytech., Palaiseau, 1995. MR 1362552 $(96 \mathrm{j}: 47049)$

Department of Mathematics and Computer Science, Southwestern University, Georgetown, Texas 78626

E-mail address: futamurf@southwestern.edu 